\title{
FACTOR GENERALIZED BE-SEMIGROUPS THROUGH HOMOMORPHISMS
}

\author{
S. SALEEM ${ }^{1}$, F. HUSSAIN ${ }^{1}$, B. DAVVAZ ${ }^{2}$ and M.T. RAHIM ${ }^{3}$ \\ 1,3 Department of Mathematics, Abbottabad University of Science and Technology, Pakistan. \\ ${ }^{2}$ Department of Mathematics Yazd University, Yazd, Iran \\ ${ }^{*}$ Corresponding author. E-mail: fawad.hussain30@gmail.com
}

\section{DOI: 10.20948/mathmontis-2021-50-6}

Summary. In this paper, we generalize the concept of homomorphism from BE-semigroups to generalized BE-semigroups. In order to show the existence, we construct some examples. Furthermore, we characterize generalized BE-semigroups by using homomorphism. In particular, we show that through every homomorphism, we may get factor generalized BEsemigroup.

\section{INTRODUCTION}

Two classes of abstract algebras had been defined by Tanaka and Iseki. These are called BCK-algebras [1] and BCI-algebras [2]. It is well known that every BCK-algebra is a BCIalgebra, i.e. in other words, BCI-algebra is a generalization of a BCK-algebra. Moreover, Neggers and Kim [3] introduced the idea of a d-algebra which is a generalized structure for a BCK-algebra. Furthermore, Jun et al. [4] defined a new class of an abstract algebra, named as a $\mathrm{BH}$-algebra. It is known that a $\mathrm{BH}$-algebra is a generalized structure of BCK and BCIalgebras. Later on, H.S. Kim and Y. H. Kim [5] introduced another generalized structure of a BCK-algebra known as a BE-algebra. The authors of [5] provided equivalent conditions for the filters in a BE-algebra and for this they used the concept of upper sets. Again in [6], Ahn and So defined ideals in BE-algebras and explored a number of associated properties of such ideals. The authors of [6,7] also discussed upper sets and generalized upper sets and characterized them by different properties. In [8], Ahn and Kim combined two structures, i.e. BE-algebra and semigroup and defined the structure of a BE-semigroup. In the research article [9], the author introduced the idea of BE-homomorphisms of BE-semigroups and characterized BE-semigroups by the properties of BE-homomorphisms. Moreover, he introduced the concept of factor self-distributive BE-semigroups and investigated some of their properties. Recently the authors of [10] have given a new generalization of a BE-algebra known as PSRU-algebra. They have discussed left (resp. right) ideal as well as filter in the same structure and investigated a relationship between left ideal and filter.

\section{PRELIMINARIES}

In this portion, we discuss generalized BE-semigroup which is a generalization of a BEsemigroup. We give some examples and discuss some of their properties. Furthermore, we discuss different classes of generalized BE-semigroups. Firstly, we are going to define generalized BE-algebra and for the definition, we refer the readers to [11].

2010 Mathematics Subject Classification: 06F35 (20M99).

Keywords and Phrases: GBE-semigroup, Congruence relation, Homomorphism. 


\section{Definition 2.1}

Let $\emptyset \neq \underline{R}$ be a set together with a binary operation " $*$ " and a constant " $1_{\underline{\underline{R}}}$ ", then it is said to be a generalized BE-algebra (shortly denoted by GBE-algebra) if the following conditions are satisfied:

(i) $\forall v \in \underline{R}, v * v=1_{\underline{R}}$,

(ii) $\forall v \in \underline{R}, v * 1_{\underline{R}}=1_{\underline{R}}$,

(iii) $\forall v, \hat{e}, w \in \underline{R}, v *(\hat{e} * w)=\hat{e} *(v * w)$.

The example which is given below shows the existence of the above structure.

\section{Example 2.2 [11]}

Let $\dot{\boldsymbol{W}}=\left\{1_{\dot{W}}, 2,3\right\}$ be a set and “*” is defined in $\dot{\boldsymbol{W}}$ in the following table.

\begin{tabular}{|c|c|c|c|}
\hline & $1_{\dot{W}}$ & 2 & 3 \\
\hline $1_{W}$ & $1_{\dot{W}}$ & $1_{W}^{\prime}$ & $1_{W}$ \\
\hline 2 & $1_{W}$ & $1_{W}$ & 3 \\
\hline 3 & $1_{\dot{W}}$ & 2 & $1_{W}$ \\
\hline
\end{tabular}

Then $\left(\dot{W} ; *, 1_{\dot{W}}^{\prime}\right)$ is a GBE-algebra.

Note that in GBE-algebra $\left(\underline{\boldsymbol{R}} ; *, 1_{\underline{\underline{R}}}\right)$, we define a relation " $\leq$ "' by $u \leq s \Leftrightarrow u * s=1_{\underline{\underline{R}}}$. Throughout this paper, we shall always assume that $u \leq s$ and $s \leq u$ implies that $u=s$. Now one can easily see that the following identities are true in a GBE-algebra.

(i) $\underline{u} *(s * u \underline{u})=1_{\underline{\underline{R}}}$,

(ii) $u *((u * s) * s)=1_{\underline{R}}$.

We now have the following definition which is taken from [11].

\section{Definition 2.3}

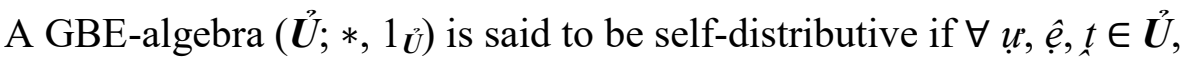

$$
u *(\hat{e} * t)=(u * \hat{e}) *(u * t) .
$$

Let us give an example in order to show the existence of a self-distributive GBE-algebra.

\section{Example 2.4 [11]}

Assume that $\dot{\boldsymbol{W}}=\left\{1_{\dot{\boldsymbol{W}}}, 2,3\right\}$ be a set and “*” is defined in $\dot{\boldsymbol{W}}$ in the following table: 


\begin{tabular}{|c|c|c|c|}
\hline$*$ & $1_{W}$ & 2 & 3 \\
\hline $1_{\tilde{W}}$ & $1_{\mathscr{W}}$ & $1_{\tilde{W}}$ & $1_{\mathscr{W}}$ \\
\hline 2 & $1_{W}$ & $1_{\dot{W}}$ & 3 \\
\hline 3 & $1_{\dot{W}}$ & 2 & $1_{\dot{W}}$ \\
\hline
\end{tabular}

Then $\boldsymbol{W}$ is a self-distributive GBE-algebra.

Further, we have the following definitions which are taken from [11].

\section{Definition 2.5}

A GBE-algebra $\left(\boldsymbol{L} ; *, 1_{\boldsymbol{L}}\right)$ is said to be transitive if for any $u, \tilde{n}, v \in \boldsymbol{L}$

$$
\tilde{n} * v \leq(u * \tilde{n}) *(\mu * v) .
$$

Note that if $\left(\boldsymbol{L} ; *, 1_{\boldsymbol{L}}\right)$ is a GBE-algebra which is self-distributive, then $\left(\boldsymbol{L} ; *, 1_{\boldsymbol{L}}\right)$ must be transitive.

\section{Definition 2.6}

Let us suppose that $(\boldsymbol{W}, *)$ and $\left(\boldsymbol{M},{ }^{*}\right)$ are two GBE-algebras. A mapping $\varphi: \boldsymbol{W} \rightarrow \boldsymbol{M}$ is said to be a homomorphism from $\boldsymbol{W}$ into $\boldsymbol{M}$ if

$$
\varphi(\hat{e} * t)=\varphi(\hat{e}) * \varphi(t) \forall \hat{e}, t \in \boldsymbol{W} .
$$

\section{Definition 2.7}

Let we have a non-empty set $\boldsymbol{K}$ along with two binary operations " $\odot$ " and “ $*$ " and a constant " $1 \boldsymbol{K}$ ", then $\boldsymbol{K}$ is known to be a GBE-semigroup if it satisfies the conditions given below:

(i) $(\boldsymbol{K} ; \odot)$ is a semigroup,

(ii) $\left(\boldsymbol{K} ; *, 1_{\boldsymbol{\kappa}}\right)$ is a GBE-algebra,

(iii) “ $\odot$ ” is distributive (left and right) over “ $*$ ”, that is,

$\hat{e} \odot(t * w)=(\hat{e} \odot t) *(\hat{e} \odot w)$ and $(\hat{e} * t) \odot w=(\hat{e} \odot w) *(t \odot w) \forall \hat{e}, t, w \in \boldsymbol{K}$.

Let us give some examples.

\section{Example 2.8 [11]}

Let $\boldsymbol{W}=\left\{1_{\boldsymbol{W}}, 2,3,4\right\}$ and define " $\odot$ " and “*” in $\boldsymbol{W}$ in the following tables: 


\begin{tabular}{|c|c|c|c|c|}
\hline$\odot$ & $1_{W}$ & 2 & 3 & 4 \\
\hline $1_{W}$ & $1_{W}$ & $1_{W}$ & $1_{W}$ & $1_{W}$ \\
\hline 2 & $1_{W}$ & $1_{W}$ & $1_{W}$ & $1_{W}$ \\
\hline 3 & $1_{W}$ & $1_{W}$ & $1_{W}$ & 2 \\
\hline 4 & $1_{W}$ & $1_{W}$ & $1_{W}$ & $1_{W}$ \\
\hline
\end{tabular}

\begin{tabular}{|c|c|c|c|c|}
\hline$*$ & $1_{W}$ & 2 & 3 & 4 \\
\hline $1_{W}$ & $1_{W}$ & $1_{W}$ & $1_{W}$ & $1_{W}$ \\
\hline 2 & $1_{W}$ & $1_{W}$ & $1_{W}$ & $1_{W}$ \\
\hline 3 & $1_{W}$ & 2 & $1_{W}$ & 2 \\
\hline 4 & $1_{W}$ & $1_{W}$ & $1_{W}$ & $1_{W}$ \\
\hline
\end{tabular}

Then $\left(\boldsymbol{W} ; \odot, *, 1_{W}\right)$ is a GBE-semigroup.

\section{Example 2.9}

Let $\vec{U}=\left\{1_{\vec{U}}, 2,3,4,5\right\}$ and define “ $\odot$ ” and “ “*” in $\overrightarrow{\boldsymbol{U}}$ in the following tables:

\begin{tabular}{|c|c|c|c|c|c|}
\hline$\odot$ & $1_{U}$ & 2 & 3 & 4 & 5 \\
\hline $1_{\vec{U}}$ & $1_{\vec{U}}$ & $1_{\vec{U}}$ & $1_{U}$ & $1_{\vec{U}}$ & $1_{\vec{U}}$ \\
\hline 2 & $1_{\vec{U}}$ & $1_{\vec{U}}$ & $1_{\vec{U}}$ & $1_{\vec{U}}$ & $1_{\vec{U}}$ \\
\hline 3 & $1_{U}$ & $1_{\vec{U}}$ & $1_{U}$ & $1_{U}$ & $1_{\vec{U}}$ \\
\hline 4 & $1_{\vec{U}}$ & $1_{\vec{U}}$ & $1_{U}$ & $1_{\vec{U}}$ & $1_{\vec{U}}$ \\
\hline 5 & $1_{U}$ & $1_{\vec{U}}$ & $1_{U}$ & $1_{U}$ & 5 \\
\hline
\end{tabular}

\begin{tabular}{|c|c|c|c|c|c|}
\hline$*$ & $1_{\dot{U}}$ & 2 & 3 & 4 & 5 \\
\hline $1_{\vec{U}}$ & $1_{\dot{U}}$ & 3 & 3 & 4 & $1_{U}$ \\
\hline 2 & $1_{\vec{U}}$ & $1_{\vec{U}}$ & $1_{\vec{U}}$ & 4 & $1_{U}$ \\
\hline 3 & $1_{\vec{U}}$ & $1_{\vec{U}}$ & $1_{\vec{U}}$ & $1_{\boldsymbol{U}}$ & 5 \\
\hline 4 & $1_{\vec{U}}$ & 2 & 3 & $1_{\vec{U}}$ & $1_{\vec{U}}$ \\
\hline 5 & $1_{\vec{U}}$ & $1_{\vec{U}}$ & $1_{\vec{U}}$ & $1_{\vec{U}}$ & $1_{U}$ \\
\hline
\end{tabular}

Then $\left(\overrightarrow{\boldsymbol{U}} ; \odot, *, 1_{\vec{U}}\right)$ is a GBE-semigroup.

Let us give some properties.

Proposition 2.10 [11]

The following are true for a GBE-semigroup $\left(\dot{\boldsymbol{W}} ; \odot, *, 1_{\dot{W}}\right)$.

(i) $1_{\dot{W}} \odot \hat{e}=\hat{e} \odot 1_{\dot{W}}=1_{\dot{W}} \forall \hat{e} \in \dot{W}$,

(ii) $\hat{e} \leq s \Rightarrow \hat{e} \odot v \leq s \odot v, v \odot \hat{e} \leq r \odot s \forall \hat{e}, s, v \in \dot{W}$. 
We are now going to define unit divisor. For the following definition we refer the readers to $[11]$.

\section{Definition 2.11}

In a GBE-semigroup $\left(\boldsymbol{Y} ; \odot, *, 1_{\boldsymbol{Y}}\right), 1_{\boldsymbol{Y}} \neq v \in \boldsymbol{Y}$ is a left unit divisor if $\exists 1_{\boldsymbol{Y}} \neq s \in \boldsymbol{Y}$ such that $v \odot s=1_{Y}$. Similarly we may define a right unit divisor.

Let $1_{\boldsymbol{Y}} \neq v \in \boldsymbol{Y}$, which is right as well as left unit divisor of $\boldsymbol{Y}$, then it is called a unit divisor of $\boldsymbol{Y}$.

\section{MAIN RESULTS THROUGH HOMOMORPHISMS}

In this section, we discuss factor GBE-semigroups through homomorphism. We show that every homomorphism defines a congruence relation on every GBE-semigroup. Once we get the said congruence relation, we shall get factor GBE-semigroup.

\section{Definition 3.1}

Let us suppose that $\left(\boldsymbol{K} ; \odot, *, 1_{\boldsymbol{K}}\right)$ and $\left(\boldsymbol{M} ; \odot,{ }^{*}, 1_{\boldsymbol{M}}\right)$ are two GBE-semigroups. A mapping $\varphi: \boldsymbol{K} \rightarrow \boldsymbol{M}$ is said to be a homomorphism if

$$
\varphi(v * s)=\varphi(v) * \varphi(s) \text { and } \varphi(v \odot s)=\varphi(v) \odot \varphi(s) \text { for all } v, s \in \boldsymbol{K} .
$$

A homomorphism $\varphi$ is called a monomorphism (resp. epimorphism) if it is one-one (resp. onto). A homomorphism which is both one-one and onto is called an isomorphism. For any homomorphism $\varphi: \boldsymbol{K} \rightarrow \boldsymbol{M}$, the set $\left\{\dot{n} \in \boldsymbol{K}: \varphi(\dot{n})=1_{\boldsymbol{M}}\right\}$ is called the kernel of $\varphi$ and is represented by the symbol $\operatorname{Ker}(\varphi)$ while the set $\{\varphi(\dot{n}): \dot{n} \in \boldsymbol{K}\}$ is known as the image of $\varphi$ and is represented by $\operatorname{Im}(\varphi)$. The set of all homomorphisms from a GBE-semigroup $\boldsymbol{K}$ to a GBE-semigroup $\boldsymbol{M}$ is denoted by $\boldsymbol{H o m}(\boldsymbol{K}, \boldsymbol{M})$.

Let us give some examples in order to show the existence of homomorphisms.

\section{Example 3.2}

1) The identity function on any GBE-semigroup is always a homomorphism. Moreover, as the identity function is always a bijective function so it follows that the identity function on any GBE-semigroup is an isomorphism.

2) Let $\boldsymbol{T}=\left\{1_{T}, 2,3,4\right\}$ and $\dot{\boldsymbol{N}}=\left\{1_{\dot{N}}, 2,3,4,5\right\}$ be the sets with the following Cayley tables: 


\begin{tabular}{|c|c|c|c|c|}
\hline$\odot$ & $1_{T}$ & 2 & 3 & 4 \\
\hline $1_{T}$ & $1_{T}$ & $1_{T}$ & $1_{T}$ & $1_{T}$ \\
\hline 2 & $1_{T}$ & $1_{T}$ & $1_{T}$ & $1_{T}$ \\
\hline 3 & $1_{T}$ & $1_{T}$ & $1_{T}$ & 2 \\
\hline 4 & $1_{T}$ & $1_{T}$ & $1_{T}$ & $1_{T}$ \\
\hline
\end{tabular}

\begin{tabular}{|c|c|c|c|c|}
\hline$*$ & $1_{T}$ & 2 & 3 & 4 \\
\hline $1_{T}$ & $1_{T}$ & $1_{T}$ & $1_{T}$ & $1_{T}$ \\
\hline 2 & $1_{T}$ & $1_{T}$ & $1_{T}$ & $1_{T}$ \\
\hline 3 & $1_{T}$ & 2 & $1_{T}$ & 2 \\
\hline 4 & $1_{T}$ & $1_{T}$ & $1_{T}$ & $1_{T}$ \\
\hline
\end{tabular}

\begin{tabular}{|c|c|c|c|c|c|}
\hline$\odot$ & $1_{\dot{N}}$ & 2 & 3 & 4 & 5 \\
\hline $1_{\dot{N}}$ & $1_{\dot{N}}$ & $1_{\dot{N}}$ & $1_{\dot{N}}$ & $1_{\dot{N}}$ & $1_{\dot{N}}$ \\
\hline 2 & $1_{\dot{N}}$ & $1_{\dot{N}}$ & $1_{\dot{N}}$ & $1_{\dot{N}}$ & $1_{\dot{N}}$ \\
\hline 3 & $1_{\dot{N}}$ & $1_{\dot{N}}$ & $1_{\dot{N}}$ & $1_{\dot{N}}$ & $1_{\dot{N}}$ \\
\hline 4 & $1_{\dot{N}}$ & $1_{\dot{N}}$ & $1_{\dot{N}}$ & $1_{\dot{N}}$ & $1_{\dot{N}}$ \\
\hline 5 & $1_{\dot{N}}$ & $1_{\dot{N}}$ & $1_{\dot{N}}$ & $1_{\dot{N}}$ & 5 \\
\hline
\end{tabular}

\begin{tabular}{|c|c|c|c|c|c|}
\hline$*$ & $1_{\dot{N}}$ & 2 & 3 & 4 & 5 \\
\hline $1_{\dot{N}}$ & $1_{\dot{N}}$ & 3 & 3 & $1_{\dot{N}}$ & $1_{\dot{N}}$ \\
\hline 2 & $1_{\dot{N}}$ & $1_{\dot{N}}$ & $1_{\dot{N}}$ & 4 & $1_{\dot{N}}$ \\
\hline 3 & $1_{\dot{N}}$ & $1_{\dot{N}}$ & $1_{\dot{N}}$ & $1_{\dot{N}}$ & 5 \\
\hline 4 & $1_{\dot{N}}$ & 2 & 3 & $1_{\dot{N}}$ & $1_{\dot{N}}$ \\
\hline 5 & $1_{\dot{N}}$ & $1_{\dot{N}}$ & $1_{\dot{N}}$ & $1_{\dot{N}}$ & $1_{\dot{N}}$ \\
\hline
\end{tabular}

Then $\left(\boldsymbol{T} ; \odot, *, 1_{\boldsymbol{T}}\right)$ and $\left(\dot{\boldsymbol{N}} ; \odot,{ }^{*}, 1_{\dot{N}}\right)$ are GBE-semigroups.

Define $\varphi: T \rightarrow \dot{\boldsymbol{N}}$ by

$$
\varphi\left(1_{T}\right)=1_{\dot{N}}, \varphi(2)=1_{\dot{N}}, \varphi(3)=1_{\dot{N}} \text { and } \varphi(4)=4 .
$$

Then we can easily check that $\varphi$ is a homomorphism from $\left(\boldsymbol{T} ; \odot, *, 1_{T}\right)$ into $\left(\dot{N} ; \odot,{ }^{*}, 1_{\dot{N}}\right)$.

Let us state and prove some properties. The properties are true in case of BE-semigroups. We convert them into GBE-semigroups.

\section{Proposition 3.3}

Let $\psi: \boldsymbol{L} \rightarrow \boldsymbol{K}$ be a homomorphism of GBE-semigroups $\left(\boldsymbol{L} ; \odot, *, 1_{\boldsymbol{L}}\right)$ and $\left(\boldsymbol{K} ; \odot,{ }^{*}, 1_{\boldsymbol{K}}\right)$. Then

(i) $\psi\left(1_{L}\right)=1_{\kappa}$,

(ii) Let $t * w=1_{\boldsymbol{L}}$ for all $t, w \in \boldsymbol{L}$, then $\psi(t) * \psi(w)=1_{\boldsymbol{K}}$. 


\section{Proof.}

Here $\psi\left(1_{\boldsymbol{L}}\right)=\psi\left(1_{\boldsymbol{L}} * 1_{\boldsymbol{L}}\right)=\psi\left(1_{\boldsymbol{L}}\right) * \psi\left(1_{\boldsymbol{L}}\right)=1_{\boldsymbol{K}}$. Therefore (i) is satisfied. Let $t, w \in \boldsymbol{L}$ and $t * w=1_{L}$. By using (i), we have $\psi(t) * \psi(w)=\psi(t * w)=\psi\left(1_{L}\right)=1_{\kappa}$.

\section{Proposition 3.4}

Assume that $\left(\underline{\boldsymbol{T}} ; \odot, *, 1_{\underline{T}}\right)$ and $\left(\boldsymbol{M} ; \odot,{ }^{*}, 1_{\boldsymbol{M}}\right)$ are GBE-semigroups. Let $\psi: \underline{\boldsymbol{T}} \rightarrow \boldsymbol{M}$ be a homomorphism. Then $\psi$ is $1-1 \Leftrightarrow \operatorname{Ker}(\psi)=\left\{1_{\underline{T}}\right\}$.

\section{Proof.}

Let $\psi: \underline{\boldsymbol{T}} \rightarrow \boldsymbol{M}$ is a monomorphism. Let $\tilde{e} \in \boldsymbol{K} \boldsymbol{\operatorname { e r }}(\psi) \Longrightarrow \psi(\tilde{e})=1_{\boldsymbol{M}} \Rightarrow \psi(\tilde{e})=\psi\left(1_{\underline{T}}\right)$, by Proposition 3.3. As $\psi$ is $1-1$, so it follows that $\tilde{e}=1_{\underline{T}}$. Thus $\operatorname{Ker}(\psi)=\left\{1_{\underline{T}}\right\}$.

Conversely, suppose that $\operatorname{Ker}(\psi)=\left\{1_{\underline{T}}\right\}$. We need to show that $\psi$ is $1-1$. For this let $\tilde{e}, v$ $\in \underline{\boldsymbol{T}}$ be such that $\psi(\tilde{e})=\psi(v) \Longrightarrow \psi(\tilde{e}) * \psi(v)=\psi(v) * \psi(v) \Rightarrow \psi(\tilde{e} * v)=1_{M} \Rightarrow \tilde{e} * v$ $\in \operatorname{Ker}(\psi)=\left\{1_{\underline{T}}\right\} \Rightarrow \tilde{e} * v=1_{\underline{T}} \Rightarrow \tilde{e} \leq v$. Similarly, again taking $\psi(\tilde{e})=\psi(v) \Rightarrow$ $\psi(\tilde{e}) * \psi(\tilde{e})=\psi(v) * \psi(\tilde{e}) \Rightarrow 1_{M}=\psi(v * \tilde{e}) \Rightarrow v * \tilde{e} \in \operatorname{Ker}(\psi)=\left\{1_{\underline{T}}\right\} \Rightarrow v * \tilde{e}=1_{\underline{T}} \Rightarrow$ $v \leq \tilde{e}$. It follows that $\tilde{e}=v \Rightarrow \psi$ is a monomorphism.

\section{Proposition 3.5}

Let us suppose that $\psi: \boldsymbol{W} \rightarrow \boldsymbol{M}$ is a monomorphism of two GBE-semigroups $\left(\boldsymbol{W} ; \odot, *, 1_{\boldsymbol{W}}\right)$ and $\left(\boldsymbol{M} ; \odot,{ }^{*}, 1_{\boldsymbol{M}}\right)$. Let $v \in \boldsymbol{W}$ be a unit divisor of $\boldsymbol{W}$. Then $\psi(v)$ is a unit divisor of $\boldsymbol{M}$.

\section{Proof.}

Suppose $1_{\boldsymbol{W}} \neq v \in \boldsymbol{W}$ is a left unit divisor of $\boldsymbol{W}$, then $\exists 1_{\boldsymbol{W}} \neq t \in \boldsymbol{W}$ such that $v \odot t=1_{\boldsymbol{W}}$ $\Longrightarrow \psi(v \odot t)=\psi\left(1_{W}\right) \Rightarrow \psi(v) \odot \psi(t)=1_{M}$, as $\psi\left(1_{W}\right)=1_{M}$ and $\psi$ is a homomorphism. It implies that $\psi(v)$ is a left unit divisor of $\boldsymbol{M}$. Similarly let $1_{\boldsymbol{W}} \neq v \in \boldsymbol{W}$ be a right unit divisor of $\boldsymbol{W}$, then $\exists 1_{W} \neq t \in \boldsymbol{W} \ni t \odot v=1_{W} \Rightarrow \psi(t \odot v)=\psi\left(1_{W}\right) \Rightarrow \psi(t) \odot \psi(v)=1_{M}$, as $\psi\left(1_{W}\right)=1_{M}$ and $\psi$ is a homomorphism. It implies that $\psi(v)$ is a right unit divisor of $\boldsymbol{M}$. This proves what we wanted.

\section{Proposition 3.6}

Suppose that $\left(\boldsymbol{L} ; \odot_{1}, *_{1}, 1_{\boldsymbol{L}}\right),\left(\boldsymbol{M} ; \odot_{2}, *_{2}, 1_{\dot{\boldsymbol{M}}}\right)$ and $\left(\dot{\boldsymbol{N}} ; \odot_{3}, *_{3}, 1_{\dot{\boldsymbol{N}}}\right)$ are GBE-semigroups. Let $\psi \in \boldsymbol{H o m}(\boldsymbol{L}, \dot{M})$ and suppose that $\beta \in \boldsymbol{H o m}(\dot{M}, \dot{N})$, then $\beta$ o $\psi \in \operatorname{Hom}(\boldsymbol{L}, \dot{N})$.

\section{Proof.}

Let $\psi: \boldsymbol{L} \rightarrow \dot{\boldsymbol{M}}$ and $\beta: \dot{\boldsymbol{M}} \rightarrow \dot{\boldsymbol{N}}$ be homomorphisms, then we show $\beta$ o $\psi: \boldsymbol{L} \rightarrow \dot{\boldsymbol{N}}$ is a homomorphism. Let $v, \hat{e} \in \boldsymbol{L}$, then

$$
\begin{aligned}
\beta \text { o } \psi\left(v *_{1} \hat{e}\right) & =\beta\left(\psi\left(v *_{1} \hat{e}\right)\right) \\
& =\beta\left(\psi(v) *_{2} \psi(\hat{e})\right) \quad(\because \psi \text { is a homomorphism })
\end{aligned}
$$




$$
\begin{aligned}
& =\beta(\psi(v)) *_{3} \beta(\psi(\hat{e})) \quad(\because \beta \text { is a homomorphism }) \\
& =\beta \text { о } \psi(v) *_{3} \beta \text { o } \psi(\hat{e}) .
\end{aligned}
$$

Similarly,

$$
\begin{aligned}
\beta \text { о } \psi\left(v \odot_{1} \hat{e}\right) & =\beta\left(\psi\left(v \odot_{1} \hat{e}\right)\right) \\
& =\beta\left(\psi(v) \odot_{2} \psi(\hat{e})\right) \quad(\because \psi \text { is a homomorphism }) \\
& =\beta(\psi(v)) \odot_{3} \beta(\psi(\hat{e}))(\because \beta \text { is a homomorphism }) \\
& =\beta \text { о } \psi(v) \odot_{3} \beta \text { о } \psi(\hat{e}) .
\end{aligned}
$$

It follows that $\beta$ o $\psi: \boldsymbol{L} \longrightarrow \dot{\boldsymbol{N}}$ is a homomorphism or in other words, $\beta$ o $\psi \in \operatorname{Hom}(\boldsymbol{L}, \dot{N})$.

\section{Theorem 3.7}

Let us assume that $\left(\boldsymbol{W} ; \odot, *, 1_{\boldsymbol{W}}\right)$ and $\left(\boldsymbol{M} ; \odot, *, 1_{\boldsymbol{M}}\right)$ are two GBE-semigroups and $\varphi \in \boldsymbol{H o m}(\boldsymbol{W}, \boldsymbol{M})$ and furthermore suppose that $\boldsymbol{W}$ is transitive, then $\varphi(\boldsymbol{W})$ is transitive.

\section{Proof.}

Let $\varphi(\hat{e}), \varphi(\dot{r}), \varphi(w) \in \varphi(\boldsymbol{W})$. Then

$$
\begin{aligned}
(\varphi(\hat{e}) * \varphi(w)) *((\varphi(\hat{e}) * \varphi(\dot{r})) *(\varphi(\hat{e}) * \varphi(w))) & =\varphi(\dot{r} * w) *(\varphi(\hat{e} * \dot{r}) * \varphi(\hat{e} * w)) \\
& =\varphi(\dot{r} * w) * \varphi((\hat{e} * \dot{r}) *(\hat{e} * w)) \\
& =\varphi((\dot{r} * w) *((\hat{e} * \dot{r}) *(\hat{e} * w))) \\
& =\varphi\left(1_{w}\right) \\
& =1_{M} .
\end{aligned}
$$

Hence, $(\varphi(\hat{e}) * \varphi(w)) \leq((\varphi(\hat{e}) * \varphi(\dot{r})) *(\varphi(\hat{e}) * \varphi(w)))$. Therefore, $\varphi(\boldsymbol{W})$ is transitive.

\section{Theorem 3.8}

Let $\left(\boldsymbol{K} ; \odot, *, 1_{\boldsymbol{K}}\right)$ and $\left(\boldsymbol{M} ; \odot,{ }^{*}, 1_{\boldsymbol{M}}\right)$ be two GBE-semigroups. Assume that $\varphi: \boldsymbol{K} \rightarrow \boldsymbol{M}$ is a monomorphism and $\varphi(\boldsymbol{K})$ is transitive, then $\boldsymbol{K}$ is transitive.

\section{Proof.}

Let us suppose that $\dot{r}, \hat{e}, v \in \boldsymbol{K}$. Then $(\varphi(\hat{e}) * \varphi(v)) *((\varphi(\dot{r}) * \varphi(\hat{e})) *(\varphi(\dot{r}) * \varphi(v)))=1_{M}$, and thus $\varphi((\hat{e} * v) *((\dot{r} * \hat{e}) *(\dot{r} * v)))=1_{M}$ implies that $\varphi((\hat{e} * v) *((\dot{r} * \hat{e}) *(\dot{r} * v)))=$ $\varphi\left(1_{\kappa}\right)$. As $\varphi$ is a monomorphism, so by Proposition $3.4,(\hat{e} * v) *((\dot{r} * \hat{e}) *(\dot{r} * v))=1_{K}$. Thus, $\boldsymbol{K}$ is transitive. 


\section{Theorem 3.9}

Suppose $\left(\boldsymbol{L} ; \odot_{1}, *_{1}, 1_{\boldsymbol{L}}\right),\left(\boldsymbol{M} ; \odot_{2}, *_{2}, 1_{\boldsymbol{M}}\right)$ and $\left(\boldsymbol{N} ; \odot_{3}, *_{3}, 1_{\boldsymbol{N}}\right)$ are GBE-semigroups. Let $p: \boldsymbol{L} \rightarrow \boldsymbol{M}$ be an epimorphism and $\bar{g}: \boldsymbol{L} \rightarrow \boldsymbol{N}$ a homomorphism. Further suppose that $\operatorname{Ker}(p) \subseteq \operatorname{Ker}(\bar{g})$, then $\exists$ one and only one homomorphism $v: M \rightarrow N \ni \vee$ o $p=\bar{g}$, i.e. in other words the diagram

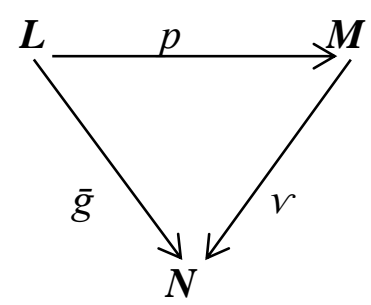

commutes.

\section{Proof.}

Let $s \in \boldsymbol{M}$. As $p$ is surjective so $\exists \dot{n} \in \boldsymbol{L} \ni p(\dot{n})=s$. Let us define a mapping

$$
\checkmark: M \rightarrow N \text { by } r(s)=v(p(\dot{n}))=\bar{g}(\dot{n}) .
$$

Well-defined: Let $w, u \in \boldsymbol{L}$. If $s=p(w)=p(u)$ then, $1_{M}=p(w) *_{2} p(u)$. Now as $p$ is a homomorphism, so it follows that $1_{M}=p\left(w *_{1} u\right)$. Hence, $w *_{1} u \in \operatorname{Ker}(p)$. As $\operatorname{Ker}(p) \subseteq$ $\operatorname{Ker}(\bar{g})$, we have $1_{N}=\bar{g}(w) *_{3} \bar{g}(\varphi)=\bar{g}\left(w *_{1} \varphi\right)$. Similarly, we get $\bar{g}(\varphi) *_{3} \bar{g}(w)=1_{N}$. Thus, it follows that $\bar{g}(w)=\bar{g}(u) \Longrightarrow v$ is well-defined. Furthermore, we prove that $v$ is a homomorphism. Now assume $\hat{e}, t \in \boldsymbol{M}$, then $\exists w, \varphi \in \boldsymbol{L}$ such that $\hat{e}=p(w)$ and $t=p(\varphi)$, as $p$ is onto. Now we have,

$$
\begin{array}{rlrl}
v\left(\hat{e} \odot_{2} t\right) & =v\left(p(w) \odot_{2} p(u)\right) & & \\
& =v\left(p\left(w \odot_{1} u\right)\right) & & (\because p \text { is a homomorphism }) \\
& =\bar{g}\left(w \odot_{1} u\right) & (\because v(p(\dot{n}))=\bar{g}(\dot{n})) \\
& =\bar{g}(w) \odot_{3} \bar{g}(u) & (\because \bar{g} \text { is a homomorphism }) \\
& =v(p(w)) \odot_{3} v(p(u)) \\
& =v(\hat{e}) \odot_{3} v(t)
\end{array}
$$

and

$$
\begin{array}{rlrl}
v\left(\hat{e} *_{2} t\right) & =v\left(p(w) *_{2} p(u)\right) & & \\
& =v\left(p\left(w *_{1} u\right)\right) & & (\because p \text { is a homomorphism }) \\
& =\bar{g}\left(w *_{1} u\right) & & (\because v(p(\dot{n}))=\bar{g}(\dot{n})) \\
& =\bar{g}(w) *_{3} \bar{g}(u) & (\because \bar{g} \text { is a homomorphism })
\end{array}
$$




$$
\begin{aligned}
& =v(p(w)) *_{3} v(p(u)) \\
& =v(\hat{e}) * *_{3} v(t) .
\end{aligned}
$$

Hence $v$ is a homomorphism.

Now

$$
\vee \circ p(\dot{n})=v(p(\dot{n}))=\bar{g}(\dot{n}) \Longrightarrow v \circ p=\bar{g}
$$

\section{Uniqueness:}

Let $v_{1}: M \rightarrow N$ be homomorphism such that $v_{1} \mathrm{o} p=\bar{g}$.

Now

$$
v_{1} \mathrm{o} p(\dot{n})=\bar{g}(\dot{n})=v \text { o } p(\dot{n}) \Rightarrow v_{1}(p(\dot{n}))=v(p(\dot{n})) \Rightarrow v_{1}(s)=v(s) \Rightarrow v_{1}=v .
$$

Hence $r$ is unique.

\section{Theorem 3.10}

Let us suppose that $\left(\boldsymbol{L} ; \odot_{1}, *_{1}, 1_{\boldsymbol{L}}\right),\left(\boldsymbol{M} ; \odot_{2}, *_{2}, 1_{\boldsymbol{M}}\right)$ and $\left(\boldsymbol{N} ; \odot_{3}, *_{3}, 1_{N}\right)$ are GBEsemigroups. Let $\psi: \boldsymbol{L} \rightarrow \boldsymbol{N}$ be a homomorphism and $\gamma: \boldsymbol{M} \rightarrow \boldsymbol{N}$ be a monomorphism. Further, suppose that $\operatorname{Im}(\psi) \subseteq \operatorname{Im}(\gamma)$ then there is one and only one homomorphism $\varphi: \boldsymbol{L} \rightarrow \boldsymbol{M}$ such that $\gamma$ o $\varphi=\psi$, i.e. the diagram

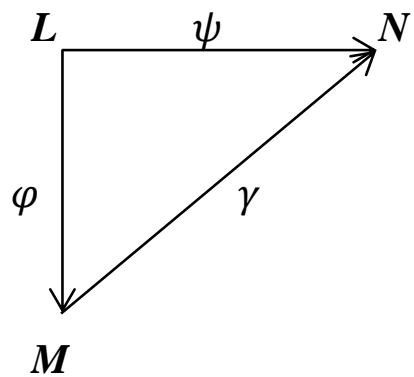

commutes.

\section{Proof.}

For $z \in \boldsymbol{L}$, then $\psi(z) \in \operatorname{Im}(\psi) \subseteq \boldsymbol{I} \boldsymbol{m}(\gamma) \Longrightarrow \psi(z) \in \operatorname{Im}(\gamma)$. Since $\gamma$ is a monomorphism so $\exists$ one and only one element $m \in \boldsymbol{M} \ni \gamma(m)=\psi(z)$. Let us define a mapping $\varphi: \boldsymbol{L} \rightarrow \boldsymbol{M}$ by $\varphi(z)=m$, then $\gamma \circ \varphi(z)=\gamma(\varphi(z))=\gamma(m)=\psi(z)$. It follows that $\gamma \circ \varphi=\psi$. Now in order to prove that $\varphi$ is a homomorphism, assume $w, s \in \boldsymbol{L}$, then

$$
\begin{aligned}
\gamma\left(\varphi\left(w *_{1} s\right)\right) & \left.=\psi\left(w *_{1} s\right)\right) & & (\text { since } \gamma \text { o } \varphi(z)=\psi(z)) \\
& =\psi(w) *_{3} \psi(s) & & (\text { since } \psi \text { is a homomorphism) } \\
& =\gamma(\varphi(w)) *_{3} \gamma(\varphi(s)) & & (\text { since } \gamma(\varphi(z))=\psi(z))
\end{aligned}
$$




$$
=\gamma\left(\varphi(w) *_{2} \varphi(s)\right) \quad \text { (since } \gamma \text { is a homomorphism) }
$$

As $\gamma$ is a one-one so we have $\varphi\left(w *_{1} s\right)=\varphi(w) *_{2} \varphi(s)$. Similarly,

$$
\begin{array}{rlr}
\gamma\left(\varphi\left(w \odot_{1} s\right)\right) & =\psi\left(w \odot_{1} s\right) & \\
& =\psi(w) \odot_{3} \psi(s) & \\
& =\gamma(\varphi(w)) \odot_{3} \gamma(\varphi(s)) & \\
& =\gamma\left(\varphi(w) \odot_{2} \varphi(s)\right) &
\end{array}
$$

As $\gamma$ is a monomorphism so we obtain $\varphi\left(w \odot_{1} s\right)=\varphi(w) \odot_{2} \varphi(s)$. Now let $\varphi_{1}: \boldsymbol{L} \rightarrow \boldsymbol{M}$ be a homomorphism such that $\gamma$ o $\varphi_{1}=\psi$. Now,

$$
\begin{aligned}
& \gamma \circ \varphi_{1}(z)=\psi(z) \\
\Rightarrow & \gamma \circ \varphi_{1}(z)=\gamma \circ \varphi(z) \\
\Rightarrow & \gamma\left(\varphi_{1}(z)\right)=\gamma(\varphi(z)) \\
\Rightarrow & \varphi_{1}(z)=\varphi(z) \quad \text { (since } \gamma \text { is a monomorphism) } \\
\Rightarrow & \varphi_{1}=\varphi .
\end{aligned}
$$

Thus, $\varphi$ is unique.

We are now going to define left (resp. right) congruence relations in a GBE-semigroup.

\section{Definition 3.11}

Let $\left(\boldsymbol{W} ; \odot, *, 1_{\boldsymbol{W}}\right)$ be a GBE-semigroup and let $\rho$ be a relation on $\boldsymbol{W}$, then $\rho$ is known to be a left compatible relation if for all $\hat{e}, \dot{n}, w \in W \ni(\hat{e}, \dot{n}) \in \rho \Rightarrow(w \odot \hat{e}, w \odot \dot{n}) \in \rho$ and $(w * \hat{e}, w * \dot{n}) \in \rho$. In the same way, let $\rho$ be a relation on a GBE-semigroup $\left(\boldsymbol{W} ; \odot, *, 1_{\boldsymbol{W}}\right)$, then $\rho$ is known to be a right compatible relation if for all $\hat{e}, \dot{n}, w \in W \ni(\hat{e}, \dot{n}) \in \rho \Rightarrow$ $(\hat{e} \odot w, \dot{n} \odot w) \in \rho$ and $(\hat{e} * w, \dot{n} * w) \in \rho$.

\section{Definition 3.12}

Let $\rho$ be a relation on a GBE-semigroup $\left(\boldsymbol{K} ; \odot, *, 1_{\boldsymbol{K}}\right)$, then $\rho$ is called compatible if for all $\hat{e}, \dot{n}, v, s \in \boldsymbol{K} \ni(\hat{e}, \dot{n}),(v, s) \in \rho \Longrightarrow(\hat{e} \odot v, \dot{n} \odot s),(\hat{e} * v, \dot{n} * s) \in \rho$.

It should be noted that if a relation is left compatible as well as equivalence relation, then it is known to be a left congruence relation. Also note that if a relation is right compatible as well as equivalence relation, then it is known to be a right congruence relation. Furthermore, note that if a relation is compatible as well as equivalence relation, then it known to be a congruence relation.

Let us give some examples in order to understand the above concepts. 


\section{Example 3.13}

(i) It is obvious that $\nabla=\boldsymbol{W} \times \boldsymbol{W}$ and $\Delta=\{(s, s): s \in \boldsymbol{W}\}$ are congruence relations on a GBE-semigroup $\left(\boldsymbol{W} ; \odot, *, 1_{\boldsymbol{W}}\right)$.

(ii) Let $\underset{\sim}{\boldsymbol{U}}=\left\{1_{\underline{U}}, 2,3,4\right\}$ and the operations “*” and " $\odot$ ” be defined as follows:

\begin{tabular}{|c|c|c|c|c|}
\hline$\odot$ & $1_{\boldsymbol{U}}$ & 2 & 3 & 4 \\
\hline $1_{\boldsymbol{U}}$ & $1_{\boldsymbol{U}}$ & $1_{\boldsymbol{U}}$ & $1_{\boldsymbol{U}}$ & $1_{\boldsymbol{U}}$ \\
\hline 2 & $1_{\boldsymbol{U}}$ & $1_{\boldsymbol{U}}$ & $1_{\boldsymbol{U}}$ & $1_{\boldsymbol{U}}$ \\
\hline 3 & $1_{\boldsymbol{U}}$ & $1_{\boldsymbol{U}}$ & $1_{\boldsymbol{U}}$ & 2 \\
\hline 4 & $1_{\boldsymbol{U}}$ & $1_{\boldsymbol{U}}$ & $1_{\boldsymbol{U}}$ & $1_{\boldsymbol{U}}$ \\
\hline
\end{tabular}

\begin{tabular}{|c|c|c|c|c|}
\hline$*$ & $1_{\boldsymbol{U}}$ & 2 & 3 & 4 \\
\hline $1_{\boldsymbol{U}}$ & $1_{\boldsymbol{U}}$ & $1_{\boldsymbol{U}}$ & $1_{\boldsymbol{U}}$ & $1_{\boldsymbol{U}}$ \\
\hline 2 & $1_{\boldsymbol{U}}$ & $1_{\boldsymbol{U}}$ & $1_{\boldsymbol{U}}$ & $1_{\boldsymbol{U}}$ \\
\hline 3 & $1_{\boldsymbol{U}}$ & 2 & $1_{\boldsymbol{U}}$ & 2 \\
\hline 4 & $1_{\boldsymbol{U}}$ & $1_{\boldsymbol{U}}$ & $1_{\boldsymbol{U}}$ & $1_{\boldsymbol{U}}$ \\
\hline
\end{tabular}

Then $\left(\boldsymbol{U} ; \odot, *, 1_{\boldsymbol{U}}\right)$ is a GBE-semigroup. Let $\theta=\Delta \cup\left\{\left(2,1_{U}\right),\left(1_{\underline{U}}, 2\right)\right\}$. Here $\theta$ is a congruence relation on $\boldsymbol{U}$.

Let us state and prove some results. The results are true in case of semigroups and we convert them into GBE-semigroups. The following result gives us equivalent conditions for congruence relations in GBE-semigroups.

\section{Proposition 3.14}

Let $\rho$ be an equivalence relation on a GBE-semigroup $\left(\boldsymbol{K} ; \odot, *, 1_{\boldsymbol{K}}\right)$. Then the following are equivalent:

(i) $\rho$ is a congruence relation on the GBE-semigroup $\boldsymbol{K}$.

(ii) $\rho$ is left and right congruence relation on the GBE-semigroup $\boldsymbol{K}$.

\section{Proof.}

(i) $\Rightarrow$ (ii) In order to prove that $\rho$ is left as well as right congruence relation, assume that $\hat{e}$, $\tilde{n}, v \in \boldsymbol{K}$ such that $(\hat{e}, \tilde{n}) \in \rho$. Now $(v, v) \in \rho$, because $\rho$ is reflexive. As $\rho$ is compatible, so it follows that $(v \odot \hat{e}, v \odot \tilde{n}),(v * \hat{e}, v * \tilde{n}) \in \rho$. It follows that $\rho$ is a left congruence relation. Similarly, we can also prove that $(\hat{e} \odot v, \tilde{n} \odot v),(\hat{e} * v, \tilde{n} * v) \in \rho$. It follows that $\rho$ is a right congruence relation.

(ii) $\Rightarrow$ (i) In order to prove that $\rho$ is a congruence relation suppose $\hat{e}, \tilde{n}, v, s \in \boldsymbol{K} \ni(\hat{e}, \tilde{n})$, $(v, s) \in \rho$. Now $(\hat{e} \odot v, \tilde{n} \odot v),(\hat{e} * v, \tilde{n} * v) \in \rho$, because $\rho$ is right compatible and $(\tilde{n} \odot v, \tilde{n} \odot s),(\tilde{n} * v, \tilde{n} * s) \in \rho$, because $\rho$ is left compatible. By transitivity, it follows that $(\hat{e} \odot v, \tilde{n} \odot s),(\hat{e} * v, \tilde{n} * s) \in \rho$. It proves what we wanted.

The below theorem confirms that every homomorphism gives us a congruence relation. 


\section{Theorem 3.15}

Suppose $\left(\boldsymbol{W} ; \odot, *, 1_{\boldsymbol{W}}\right)$ and $\left(\boldsymbol{T} ; \odot,{ }^{*}, 1_{\boldsymbol{T}}\right)$ are two GBE-semigroups. Let $\check{h}: \boldsymbol{W} \rightarrow \boldsymbol{T}$ be a homomorphism. Then $\check{h}$ defines a congruence relation $\delta$ on $\boldsymbol{W}$ as follows:

$$
\delta=\{(s, v) \in \boldsymbol{W} \times \boldsymbol{W}: \check{h}(s)=\check{h}(v)\} .
$$

\section{Proof.}

In order to prove that $\check{h}$ defines the above congruence relation $\delta$ on $\boldsymbol{W}$, we need to prove that $\delta$ is a compatible equivalence relation on $\boldsymbol{W}$. First, we prove that $\delta$ is an equivalence relation on $\boldsymbol{W}$.

\section{Reflexive:}

As $\check{h}(s)=\check{h}(s) \forall s \in \boldsymbol{W}$, so it follows that $(s, s) \in \delta \forall s \in \boldsymbol{W}$. Thus $\delta$ is reflexive.

\section{Symmetric:}

Assume $s, v \in \boldsymbol{W} \ni(s, v) \in \delta \Rightarrow \check{h}(s)=\check{h}(v) \Rightarrow \check{h}(v)=\check{h}(s) \Rightarrow(v, s) \in \delta$. Thus $\delta$ is symmetric.

\section{Transitive:}

Let $s, v, u \in \boldsymbol{W}$ such that $(s, v),(v, u) \in \delta \Rightarrow \check{h}(s)=\check{h}(v)$ and $\check{h}(v)=\check{h}(u)$. By transitive property of equality, it follows that $\check{h}(s)=\check{h}(u) \Longrightarrow(s, u) \in \delta$. Thus $\delta$ is transitive.

We now show that $\delta$ is a compatible relation on $\boldsymbol{W}$. Assume that $s, v, u, \hat{e} \in \boldsymbol{W} \ni(s, v)$, $(u, \hat{e}) \in \delta \Rightarrow \check{h}(s)=\check{h}(v)$ and $\check{h}(u)=\check{h}(\hat{e})$.

Now,

$$
\begin{gathered}
\check{h}(s) \odot \check{h}(u)=\check{h}(v) \odot \check{h}(\hat{e}) \text { and } \check{h}(s) * \check{h}(u)=\check{h}(v) * \check{h}(\hat{e}) \\
\Rightarrow \check{h}(s \odot u)=\check{h}(v \odot \hat{e}) \text { and } \check{h}(s * u)=\check{h}(v * \hat{e}) \\
\Rightarrow(s \odot u, v \odot \hat{e}) \text { and }(s * u, v * \hat{e}) \in \delta .
\end{gathered}
$$

Hence, it follows that $\delta$ is a compatible relation on $\boldsymbol{W}$. This completes the proof.

We are now going to define congruence class and then discuss factor GBE-semigroup.

\section{Definition 3.16}

Let $\left(\boldsymbol{W} ; \odot, *, 1_{W}\right)$ be a GBE-semigroup. If $\delta$ is a congruence relation on $\boldsymbol{W}$ then we define for $u \in W$

$$
\text { uc } \delta=\{s \in \boldsymbol{W}:(s, u) \in \delta\}
$$

which is called a congruence class corresponding to an element $u$. Let $\boldsymbol{W} / \delta=$ $\{u \delta: \psi \in W\}$, that is $\boldsymbol{W} / \delta$ consists of all congruence classes corresponding to the elements of 
$\boldsymbol{W}$. Our aim is to show that $\boldsymbol{W} / \delta$ is a GBE-semigroup. For this, we define " $\odot$ " and "*” on $W / \delta$ as follows:

$$
\mu \delta \odot s \delta=(u \odot s) \delta
$$

and

$$
u{ }^{*} \delta \delta=(u * s) \delta \forall \underline{\varphi} \delta, s \delta \in \boldsymbol{W} / \delta \text {. }
$$

\section{Well-defined:}

Assume $v_{1} \delta, v_{2} \delta, \dot{s}_{1} \delta, \dot{s}_{2} \delta \in \boldsymbol{W} / \delta$ such that

$$
\begin{aligned}
& v_{1} \delta=v_{2} \delta \text { and } \dot{s}_{1} \delta=\dot{s}_{2} \delta \\
\Rightarrow & \left(v_{1}, v_{2}\right) \in \delta \text { and }\left(\dot{s}_{1}, \dot{s}_{2}\right) \in \delta \\
\Rightarrow & \left(v_{1} \odot \dot{s}_{1}, v_{2} \odot \dot{s}_{2}\right) \text { and }\left(v_{1} * \dot{s}_{1}, v_{2} * \dot{s}_{2}\right) \in \delta \quad(\because \delta \text { is a congruence relation }) \\
\Rightarrow & \left(v_{1} \odot \dot{s}_{1}\right) \delta=\left(v_{2} \odot \dot{s}_{2}\right) \delta \text { and }\left(v_{1} * \dot{s}_{1}\right) \delta=\left(v_{2} * \dot{s}_{2}\right) \delta \\
\Rightarrow & v_{1} \delta \odot \dot{s}_{1} \delta=v_{2} \delta \odot \dot{s}_{2} \delta \text { and } v_{1} \delta * \dot{s}_{1} \delta=v_{2} \delta * \dot{s}_{2} \delta .
\end{aligned}
$$

We now prove that $(\boldsymbol{W} / \delta, \odot)$ is a semigroup.

\section{Closure property:}

It is clear from the definition.

\section{Associative property:}

Let $\dot{r} \delta, v \delta, t \delta \in \boldsymbol{W} / \delta$, then

$$
\begin{aligned}
(\dot{r} \delta \odot v \delta) \odot t \delta & =(\dot{r} \odot v) \delta \odot t \delta \\
& =((\dot{r} \odot v) \odot t) \delta \\
& =(\dot{r} \odot(v \odot t)) \delta \quad(\because \boldsymbol{W} \text { is a GBE-semigroup }) \\
& =\dot{r} \delta \odot(v \odot t) \delta \\
& =\dot{r} \delta \odot\left(v \delta \odot_{\lambda} \delta\right) .
\end{aligned}
$$

Thus associative property holds under " $\odot$ ". Thus, $(\boldsymbol{W} / \delta, \odot)$ is a semigroup.

We now show that $\left(\boldsymbol{W} / \delta ; *, 1_{W} \delta\right)$ is a GBE-algebra. For this we have

(i) $v \delta * v \delta=(v * v) \delta=1_{W} \delta$ for all $v \delta \in \boldsymbol{W} / \delta$.

(ii) $v \delta * 1_{W} \delta=\left(v * 1_{W}\right) \delta=1_{W} \delta$ for all $v \delta \in \boldsymbol{W} / \delta$.

(iii) $v \delta *(u \delta * \hat{e} \delta)=v \delta *(u * \hat{e}) \delta$ 


$$
\begin{aligned}
& =(v *(u * \hat{e})) \delta \\
& =(u *(v * \hat{e})) \delta \quad(\because \boldsymbol{W} \text { is a GBE-semigroup }) \\
& =u \dot{ } \delta *(v * \hat{e}) \delta
\end{aligned}
$$

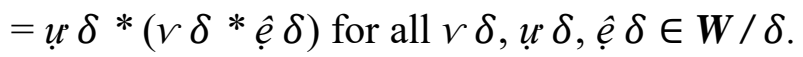

We now show that distributive laws hold in $\boldsymbol{W} / \delta$. For this, let $\tilde{e} \delta, w \delta, \dot{n} \delta \in \boldsymbol{W} / \delta$, then

$$
\begin{aligned}
\tilde{e} \delta \odot(w \delta * \dot{n} \delta) & =\tilde{e} \delta \odot(w * \dot{n}) \delta \\
& =(\tilde{e} \odot(w * \dot{n})) \delta \\
& =((\tilde{e} \odot w) *(\tilde{e} \odot \dot{n})) \delta \quad(\because \boldsymbol{W} \text { is a GBE-semigroup }) \\
& =(\tilde{e} \odot w) \delta *(\tilde{e} \odot \dot{n}) \delta \\
& =(\tilde{e} \delta \odot w \delta) *(\tilde{e} \delta \odot \dot{n} \delta) .
\end{aligned}
$$

Also

$$
\begin{aligned}
(\tilde{e} \delta * w \delta) \odot \dot{n} \delta & =(\tilde{e} * w) \delta \odot \dot{n} \delta \\
& =((\tilde{e} * w) \odot \dot{n}) \delta \\
& =((\tilde{e} \odot \dot{n}) *(w \odot \dot{n})) \delta \quad(\because \boldsymbol{W} \text { is a GBE-semigroup }) \\
& =(\tilde{e} \odot \dot{n}) \delta *(w \odot \dot{n}) \delta \\
& =(\tilde{e} \delta \odot \dot{n} \delta) *(w \delta \odot \dot{n} \delta) .
\end{aligned}
$$

The above calculation shows $\left(\boldsymbol{W} / \delta, \odot,{ }^{*}, 1_{W} \delta\right)$ is a GBE-semigroup and is called quotient or factor GBE-semigroup.

The following results give us some properties of quotient GBE-semigroups.

\section{Theorem 3.17}

Let $\delta$ be a congruence relation on a GBE-semigroup $\left(\dot{\boldsymbol{W}} ; \bigodot_{1}, *_{1}, 1_{\dot{\boldsymbol{W}}}\right)$. Then $\dot{\boldsymbol{W}} / \delta$ is a GBEsemigroup with respect to the following binary operations.

(i) $\dot{w} \delta \odot_{1} S=\left(\dot{w} \odot_{1} S\right) \delta$,

(ii) $\dot{w} \delta *_{1} \delta=\left(\dot{w} *_{1} S\right) \delta \forall \dot{w} \delta, s \delta \in \dot{W} / \delta$.

Let us define $\delta^{\#}: \dot{\boldsymbol{W}} \rightarrow \dot{\boldsymbol{W}} / \delta$ by $\delta^{\#}\left(\boldsymbol{w}^{\prime}\right)=\dot{w} \delta \forall \dot{w} \in \dot{\boldsymbol{W}}^{\prime}$, then $\delta^{\#}$ is an epimorphism. Let $\psi: \dot{\boldsymbol{W}} \rightarrow \boldsymbol{T}$ be a homomorphism, where $\left(\dot{\boldsymbol{W}} ; \odot_{1}, *_{1}, 1_{\dot{W}}\right)$ and $\left(\boldsymbol{T} ; \odot_{2}, *_{2}, 1_{\boldsymbol{T}}\right)$ are GBEsemigroups, then the relation

$$
\operatorname{Ker} \psi=\{(\dot{w}, s) \in \dot{\boldsymbol{W}} \times \dot{\boldsymbol{W}}: \psi(\dot{w})=\psi(s)\}
$$


is a congruence relation on $\dot{\boldsymbol{W}}$. Moreover, there is a monomorphism $\beta: \dot{\boldsymbol{W}} / \boldsymbol{K e r} \psi \rightarrow \boldsymbol{T} \ni$ $\operatorname{Im}(\beta)=\operatorname{Im}(\psi)$ and $\beta(\operatorname{Ker} \psi)^{\#}=\psi$.

\section{Proof.}

$\boldsymbol{W}^{\prime} / \delta$ is a GBE-semigroup with respect to the binary operation " $\odot$ " and "*" and it is clear from the above discussion. We now prove that $\delta^{\#}: \dot{W} \rightarrow \dot{W} / \delta$ is an epimorphism.

\section{Well-defined:}

Let $\hat{e}, v \in \dot{W}$ be $\ni \hat{e}=v \Rightarrow \hat{e} \delta=v \delta \Rightarrow \delta^{\#}(\hat{e})=\delta^{\#}(v) \Rightarrow \delta^{\#}$ is well-defined.

\section{Homomorphism:}

Let $w_{1}, w_{2} \in \dot{W}$, then

$$
\begin{aligned}
\delta^{\#}\left(w_{1} \bigodot_{1} w_{2}\right) & =\left(w_{1} \bigodot_{1} w_{2}\right) \delta \\
& =w_{1} \delta \odot_{1} w_{2} \delta \\
& =\delta^{\#}\left(w_{1}\right) \odot_{1} \delta^{\#}\left(w_{2}\right)
\end{aligned}
$$

and

$$
\begin{aligned}
\delta^{\#}\left(w_{1} *_{1} w_{2}\right) & =\left(w_{1} *_{1} w_{2}\right) \delta \\
& =w_{1} \delta *_{1} w_{2} \delta \\
& =\delta^{\#}\left(w_{1}\right) *_{1} \delta^{\#}\left(w_{2}\right) .
\end{aligned}
$$

It follows that, $\delta^{\#}$ is a homomorphism.

\section{Onto:}

Clearly $\delta^{\#}$ is onto, because for each $\dot{w}^{\delta} \in \dot{W}^{\prime} / \delta$ there exists $\dot{w} \in \dot{W}^{\prime}$ such that $\delta^{\#}(\dot{w})=\dot{w} \delta$. Thus, $\delta^{\#}$ is an epimorphism.

The relation $\operatorname{Ker} \psi=\left\{(\dot{w}, s) \in \dot{\boldsymbol{W}} \times \dot{\boldsymbol{W}}^{\prime}: \psi(\dot{w})=\psi(s)\right\}$ is a congruence relation because of Theorem 3.15 .

Now define, $\beta: \dot{\boldsymbol{W}} / \boldsymbol{K e r} \psi \rightarrow \boldsymbol{T}$ by $\beta(w \operatorname{Ker} \psi)=\psi(w) \forall w \boldsymbol{K e r} \psi \in \dot{\boldsymbol{W}} / \boldsymbol{K e r} \psi$. First we show that the defined map is a monomorphism. For this,

\section{Well-defined:}

Let $w_{1} \operatorname{Ker} \psi, w_{2} \operatorname{Ker} \psi \in \dot{W} / \boldsymbol{K e r} \psi$ be such that

$$
\begin{aligned}
& w_{1} \operatorname{Ker} \psi=w_{2} \operatorname{Ker} \psi \\
& \Rightarrow\left(w_{1}, w_{2}\right) \in \operatorname{Ker} \psi
\end{aligned}
$$




$$
\begin{aligned}
& \Rightarrow \psi\left(w_{1}\right)=\psi\left(w_{2}\right) \\
& \Rightarrow \beta\left(w_{1} \operatorname{Ker} \psi\right)=\beta\left(w_{2} \operatorname{Ker} \psi\right) .
\end{aligned}
$$

Thus, $\beta$ is well-defined.

\section{Homomorphism:}

Let $v \operatorname{Ker} \psi, \hat{e} \operatorname{Ker} \psi \in \dot{\boldsymbol{W}} / \operatorname{Ker} \psi$, then

$$
\begin{aligned}
\beta\left(v \operatorname{Ker} \psi *_{2} \hat{e} \operatorname{Ker} \psi\right) & =\beta\left(\left(v *_{1} \hat{e}\right) \operatorname{Ker} \psi\right) \\
& =\psi\left(v *_{1} \hat{e}\right) \\
& =\psi(v) *_{2} \psi(\hat{e}) \quad(\because \psi \text { is a homomorphism }) \\
& =\beta(v \operatorname{Ker} \psi) *_{2} \beta(\hat{e} \operatorname{Ker} \psi)
\end{aligned}
$$

and

$$
\begin{aligned}
\beta\left(v \operatorname{Ker} \psi \odot_{2} \hat{e} \operatorname{Ker} \psi\right) & =\beta\left(\left(v \odot_{1} \hat{e}\right) \operatorname{Ker} \psi\right) \\
& =\psi\left(v \odot_{1} \hat{e}\right) \\
& =\psi(v) \odot_{2} \psi(\hat{e}) \quad(\because \psi \text { is a homomorphism }) \\
& =\beta(v \operatorname{Ker} \psi) \odot_{2} \beta(\hat{e} \operatorname{Ker} \psi)
\end{aligned}
$$

Thus, $\beta$ is a homomorphism.

\section{One-One:}

Let $u_{1} \operatorname{Ker} \psi, u_{2} \operatorname{Ker} \psi \in \dot{W} / \operatorname{Ker} \psi$ be such that

$$
\begin{gathered}
\beta\left(u_{1} \operatorname{Ker} \psi\right)=\beta\left(u_{2} \operatorname{Ker} \psi\right) \\
\Rightarrow \psi\left(u_{1}\right)=\psi\left(u_{2}\right) \\
\Rightarrow\left(u_{1}, u_{2}\right) \in \operatorname{Ker} \psi \\
\Rightarrow u_{1} \operatorname{Ker} \psi=u_{2} \operatorname{Ker} \psi .
\end{gathered}
$$

Thus, $\beta$ is one-one. It follows that $\beta$ is a monomorphism. We now prove $\operatorname{Im}(\beta)=\operatorname{Im}(\psi)$.

Here,

$$
\operatorname{Im}(\psi)=\{\psi(w): w \in \dot{W}\}=\{\beta(w \operatorname{Ker} \psi): w \operatorname{Ker} \psi \in \dot{W} / \operatorname{Ker} \psi\}=\operatorname{Im}(\beta) .
$$

At the end, we show that $\beta(\operatorname{Ker} \psi)^{\#}=\psi$. In other words, $\left(\beta(\operatorname{Ker} \psi)^{\#}\right)(u)=\psi(u) \forall u \in \dot{\boldsymbol{W}}^{\prime}$. Here, 


$$
\left(\beta(\operatorname{Ker} \psi)^{\#}\right)(u)=\beta\left((\operatorname{Ker} \psi)^{\#}(u)\right)=\beta(u \operatorname{Ker} \psi)=\psi(u) .
$$

\section{Theorem 3.18}

Let $\rho$ be a congruence relation on a GBE-semigroup $\left(\boldsymbol{V} ; \odot_{1}, *_{1}, 1_{V}\right)$ and let $\varphi: \boldsymbol{V} \rightarrow \boldsymbol{T}$ be a monomorphism from $\left(\boldsymbol{V} ; \odot_{1}, *_{1}, 1_{V}\right)$ to $\left(\boldsymbol{T} ; \odot_{2}, *_{2}, 1_{T}\right)$ such that $\rho \subseteq \operatorname{Ker} \varphi$. Then there is a unique homomorphism $\beta: V / \rho \rightarrow T \ni \operatorname{Im}(\beta)=\operatorname{Im}(\varphi)$ and $\beta$ o $\rho^{\#}=\varphi$.

\section{Proof.}

Define $\beta: \boldsymbol{V} / \rho \rightarrow \boldsymbol{T}$ as follows:

$$
\beta(v \rho)=\varphi(v) \forall v \rho \in V / \rho .
$$

\section{Well-defined:}

Assume that $\dot{w} \rho, s \rho \in V / \rho$ are such that

$$
\begin{aligned}
& \dot{w} \rho=s \rho \\
\Rightarrow & (\dot{w}, s) \in \rho \subseteq \operatorname{Ker} \varphi \\
\Rightarrow & (\dot{w}, s) \in \operatorname{Ker} \varphi \\
\Rightarrow & \varphi(\dot{w})=\varphi(s) \\
\Rightarrow & \beta(\dot{w} \rho)=\beta(s \rho) .
\end{aligned}
$$

Thus, $\beta$ is well-defined.

\section{Homomorphism:}

Let $u \rho, \hat{e} \rho \in V / \rho$ then,

$$
\begin{aligned}
\beta(u \rho * \hat{e} \rho) & =\beta\left(\left(u *_{1} \hat{e}\right) \rho\right) \\
& =\varphi\left(u *_{1} \hat{e}\right) \\
& =\varphi(u) *_{2} \varphi(\hat{e}) \quad(\because \varphi \text { is a homomorphism }) \\
& =\beta(u \rho) *_{2} \beta(\hat{e} \rho)
\end{aligned}
$$

and

$$
\begin{aligned}
\beta(u \rho \odot \hat{e} \rho) & =\beta\left(\left(u \odot_{1} \hat{e}\right) \rho\right) \\
& =\varphi\left(u \odot_{1} \hat{e}\right) \\
& =\varphi(u) \odot_{2} \varphi(\hat{e}) \quad(\because \varphi \text { is a homomorphism }) \\
& =\beta(u \rho) \odot_{2} \beta(\hat{e} \rho) .
\end{aligned}
$$


Thus, $\beta$ is a homomorphism. We now prove $\operatorname{Im}(\beta)=\operatorname{Im}(\varphi)$.

Here,

$$
\operatorname{Im}(\varphi)=\{\varphi(v): v \in V\}=\{\beta(v \rho): v \rho \in V / \rho\}=\operatorname{Im}(\beta) .
$$

We now show that $\beta$ o $\rho^{\#}=\varphi$. In other words, $\left(\beta \rho^{\#}\right)(v)=\varphi(v) \forall v \in V$. Here

$$
\left(\beta \rho^{\#}\right)(v)=\beta\left(\rho^{\#}(v)\right)=\beta(v \rho)=\varphi(v) .
$$

\section{Uniqueness:}

Let $\beta_{1}: \boldsymbol{V} / \rho \rightarrow \boldsymbol{T}$ be a homomorphism such that $\beta_{1} \rho^{\#}=\varphi$.

Now,

$$
\beta_{1} \rho^{\#}(v)=\beta_{1}\left(\rho^{\#}(v)\right)=\beta_{1}(v \rho)=\varphi(v)=\beta \rho^{\#}(v)=\beta(v \rho) .
$$

It follows that $\beta_{1}(v \rho)=\beta(v \rho)$ and so $\beta_{1}=\beta$.

\section{Theorem 3.19} Then

Let $\rho, \sigma$ be congruence relations on a GBE-semigroup $\left(\boldsymbol{W} ; \odot, *, 1_{W}\right)$ such that $\rho \subseteq \sigma$.

$$
\sigma / \rho=\{(u \rho, \hat{e} \rho) \in \boldsymbol{W} / \rho \times \boldsymbol{W} / \rho:(u, \hat{e}) \in \sigma\}
$$

is a congruence relation on $\boldsymbol{W} / \rho$ and $(\boldsymbol{W} / \rho) /(\sigma / \rho) \cong \boldsymbol{W} / \sigma$.

\section{Proof.}

First we show that $\sigma / \rho$ is a congruence relation on $\boldsymbol{W} / \rho$.

\section{Reflexive:}

As $(u, u) \in \sigma \forall \psi \in \in \mathbb{W}$, because $\sigma$ is reflexive, so it follows that $(u \rho, u \rho) \in \sigma / \rho$. Thus, $\sigma / \rho$ is reflexive.

\section{Symmetric:}

Take $u \rho, \hat{e} \rho \in W / \rho \ni(u \rho, \hat{e} \rho) \in \sigma / \rho \Rightarrow(u, \hat{e}) \in \sigma \Rightarrow(\hat{e}, u) \in \sigma$, because $\sigma$ is symmetric. Thus, $(\hat{e} \rho, u \rho) \in \sigma / \rho$. This implies that $\sigma / \rho$ is symmetric.

\section{Transitive:}

Let $u \rho, \hat{e} \rho, v \rho \in W / \rho$ be such that $(u \rho, \hat{e} \rho),(\hat{e} \rho, v \rho) \in \sigma / \rho \Rightarrow(u, \hat{e}),(\hat{e}, v) \in \sigma \Rightarrow$ $(u, v) \in \sigma$, because $\sigma$ is transitive. Thus, $(u \rho, v \rho) \in \sigma / \rho$ and so $\sigma / \rho$ is transitive.

It follows that $\sigma / \rho$ is an equivalence relation. 


\section{For compatibility:}

Let $u \rho, \hat{e} \rho, v \rho, \tilde{n} \rho \in W / \rho$ be such that $(u \rho, \hat{e} \rho),(v \rho, \tilde{n} \rho) \in \sigma / \rho \Rightarrow(u, \hat{e}),(v, \tilde{n}) \in \sigma$ $\Rightarrow(u \odot v, \hat{e} \odot \tilde{n}),(u * v, \hat{e} * \tilde{n}) \in \sigma$, because $\sigma$ is compatible. Thus, it follows that

$$
((u \odot v) \rho,(\hat{e} \odot \tilde{n}) \rho),((u * v) \rho,(\hat{e} * \tilde{n}) \rho) \in \sigma / \rho .
$$

In other words,

$$
\left(u \rho \odot_{1} v \rho, \hat{e} \rho \odot_{1} \tilde{n} \rho\right),\left(u \rho *_{1} v \rho, \hat{e} \rho *_{1} \tilde{n} \rho\right) \in \sigma / \rho .
$$

It follows that $\sigma / \rho$ is a compatible relation. Hence, $\sigma / \rho$ is a congruence relation.

Define $\varphi: \boldsymbol{W} / \rho \rightarrow \boldsymbol{W} / \sigma$ by $\varphi(u \rho)=u \sigma \sigma \forall \psi \rho \rho \in \boldsymbol{W} / \rho$.

\section{Well-defined:}

Suppose $w_{1} \rho, w_{2} \rho \in \boldsymbol{W} / \rho \ni$

$$
\begin{aligned}
& w_{1} \rho=w_{2} \rho \\
\Rightarrow & \left(w_{1}, w_{2}\right) \in \rho \\
\Rightarrow & \left(w_{1}, w_{2}\right) \in \rho \subseteq \sigma \\
\Rightarrow & \left(w_{1}, w_{2}\right) \in \sigma \\
\Rightarrow & w_{1} \sigma=w_{2} \sigma \\
\Rightarrow & \varphi\left(w_{1} \rho\right)=\varphi\left(w_{2} \rho\right) .
\end{aligned}
$$

Thus, $\varphi$ is well-defined.

\section{Homomorphism:}

Let $\dot{w} \rho, s \rho \in \boldsymbol{W} / \rho$ then,

$$
\begin{aligned}
\varphi\left(\dot{w} \rho *_{1} s \rho\right) & =\varphi((\dot{w} * s) \rho) \\
& =(\dot{w} * s) \sigma \\
& =\dot{w} \sigma *_{2} s \sigma \\
& =\varphi(\dot{w} \rho) *_{2} \varphi(s \rho) .
\end{aligned}
$$

Also,

$$
\begin{aligned}
\varphi\left(\dot{w} \rho \odot_{1} s \rho\right) & =\varphi((\dot{w} \odot s) \rho) \\
& =(\dot{w} \odot s) \sigma \\
& =\dot{w} \sigma \odot_{2} s \sigma
\end{aligned}
$$




$$
=\varphi(\dot{w} \rho) \odot_{2} \varphi(s \rho) .
$$

Thus, $\varphi$ is a homomorphism. Now by Theorem 3.17, there is a monomorphism $:(\boldsymbol{W} / \rho) / \operatorname{Ker} \varphi \rightarrow \boldsymbol{W} / \sigma$ which may be defined by

$$
(u \rho(\operatorname{Ker} \varphi))=u \sigma \forall \forall \rho(\operatorname{Ker} \varphi) \in(\boldsymbol{W} / \rho) / \operatorname{Ker} \varphi .
$$

\section{Onto:}

As for each $\varphi \sigma \in W / \sigma \exists u \rho(\operatorname{Ker} \varphi) \in(\boldsymbol{W} / \rho) / \operatorname{Ker} \varphi$ such that $\beta(u \rho(\operatorname{Ker} \varphi))=\psi r \sigma$, so it follows that $\beta$ is onto. Thus, $(\boldsymbol{W} / \rho) / \operatorname{Ker} \varphi \cong \boldsymbol{W} / \sigma$. We now show that $\operatorname{Ker} \varphi=\sigma / \rho$.

Here,

$$
\begin{aligned}
\operatorname{Ker} \varphi & =\{(u \rho, \hat{e} \rho) \in \boldsymbol{W} / \rho \times \boldsymbol{W} / \rho: \varphi(u \rho)=\varphi(\hat{e} \rho)\} \\
& =\{(u \rho, \hat{e} \rho) \in \boldsymbol{W} / \rho \times \boldsymbol{W} / \rho: u \sigma=\hat{e} \sigma)\} \\
& =\{(u \rho, \hat{e} \rho) \in \boldsymbol{W} / \rho \times \boldsymbol{W} / \rho:(u, \hat{e}) \in \sigma\} \\
& =\sigma / \rho .
\end{aligned}
$$

Thus, $(\boldsymbol{W} / \rho) /(\sigma / \rho) \cong \boldsymbol{W} / \sigma$.

\section{CONCLUSION}

In this paper, a homomorphism between two generalized BE-semigroups has been defined with some non-trivial examples. Further, it has been shown that each such homomorphism defines a congruence relation. The congruence relation has been utilized to obtain quotient generalized BE-semigroups. Properties analogous to first, second and third isomorphism theorems have been explored. Results discussed in this paper have applications in different fields of mathematics and computer science for defining and developing various algebraic structures.

\section{REFERENCES}

[1] K. Is' eki and S. Tanaka, "An introduction to the theory of BCK-algebras", Mathematica Japonica, 23, 1-26 (1978).

[2] K. Is'eki, “On BCI-algebras”, Mathematics Seminar Notes, 8 (1), 125-130 (1980).

[3] J. Neggers and H.S. Kim, "On d-algebras", Mathematica Slovaca, 49, 19-26 (1999).

[4] Y.B. Jun, E.H. Roh and H.S. Kim, "On BH-algebras", Scientiae Mathematicae Japonicae Online, 1, 347-354 (1998).

[5] H.S. Kim and Y. H. Kim, “On BE-algebras”, Scientiae Mathematicae Japonicae Online, 2006 (e), 1299-1302 (2006).

[6] S.S. Ahn and K.S. So, "On ideals and upper sets in BE-algebras", Scientiae Mathematicae Japonicae, 68 (2), 279-285 (2008).

[7] S.S. Ahn and K.S. So, "On generalized upper sets in BE-algebras", Bulletin of the Korean Mathematical Society, 46 (2), 281-287 (2009).

[8] S.S. Ahn and Y.H. Kim, "On BE-semigroups", International Journal of Mathematics and Mathematical Sciences, 2011, Article ID 676020, 8 pages (2011). 
[9] A.H. Handam, “On BE-homomorphisms of BE-semigroups", International Journal of Pure and Applied Mathematics, 78 (8), 1211-1220 (2012).

[10] P. Yiarayong and P. Wachirawongsakorn, "A new generalization of BE-algebras", Heliyon, 4, Article ID e00863, 17 pages (2018).

[11] Javeria, Generalized BE-semigroups, M.Phil. Thesis, Abbottabad University of Science and Technology, Abbottabad, Pakistan, (2019).

Received February 18, 2020 